\title{
PHI-STABLE OPERATORS AND INNER APPROXIMATION-SOLVABILITY
}

\author{
RAM U. VERMA
}

(Communicated by Palle E. T. Jorgensen)

\begin{abstract}
We extend, by applying a theorem of Petryshyn (1970), the approximation-solvability of the nonlinear functional equations involving strongly stable Hilbert space mappings to the case of strongly $\phi$-stable mappings-a new and rather general class of mappings. These mappings constitute a generalization of monotone mappings. Finally, we upgrade the obtained results to the case of Banach space mappings.
\end{abstract}

\section{INTRODUCTION}

Let $X$ and $Y$ be separable complex Banach spaces with $X^{*}$ and $Y^{*}$ denoting their respective duals. Let $D$ be a given set in $X$ and $A: D \subseteq X \rightarrow Y$ be a (nonlinear) mapping. To investigate the approximation-solvability of the equation $A x=b \quad(x \in D, b \in Y)$, Petryshyn [7] and Browder and Petryshyn [1] considered an approximation scheme $\pi_{0}=\left\{X_{n}, P_{n}, Y_{n}, Q_{n}\right\}$ represented by an accompanying diagram

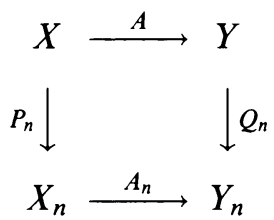

where $\left\{X_{n}\right\} \subseteq X$ and $\left\{Y_{n}\right\} \subseteq Y$ are sequences of monotonically increasing finite-dimensional subspaces with $\operatorname{dim} X_{n}=\operatorname{dim} Y_{n}$ for each $n$ and $P_{n}: X \rightarrow$ $X_{n}$ and $Q_{n}: Y \rightarrow Y_{n}$ are linear continuous projections such that $P_{n} x \rightarrow x$ and $Q_{n} y \rightarrow y$ for $x \in X$ and $y \in Y$. Here and in what follows, the symbols " $\rightarrow$ " and " $\rightarrow$ " shall denote the strong and weak convergence, respectively.

There are various numerical methods that lie beyond the scope of the approximation scheme $\pi_{0}=\left\{X_{n}, P_{n}, Y_{n}, Q_{n}\right\}$, especially if we want to approximate solutions of an equation in some function space $X$ by solutions of equations in some discrete space $X_{n}$, which is not a subspace of $X$. Motivated by these practial considerations, the upgrades of the scheme $\pi_{0}$ were considered; for example, the approximation scheme $\pi_{1}=\left\{X_{n}, E_{n}, R_{n}, Y_{n}, Q_{n}\right\}$ represented by diagram (1.2) where we embed the elements of $X_{n}$ somehow into $X$, say

Received by the editors June 3, 1991.

1991 Mathematics Subject Classification. Primary 65J15. 
by $E_{n}: X_{n} \rightarrow X$, while restricting the elements of $X$ to some $X_{n}$, say by $R_{n}: X \rightarrow X_{n}$,

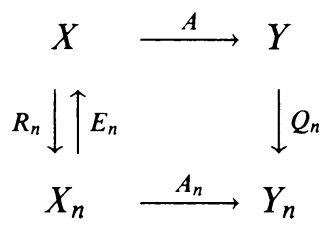

where all $A_{n}=Q_{n} A E_{n}$ are continuous. For a nice survey on the approximation schemes, see [1,2], and for more details on the approximation-solvability, see $[1,2,4,6-8,10,13-15]$.

Now we give an example where the approximation scheme $\pi_{1}$ is suitable, i.e., we want to approximate solutions of an equation in some function space $X$ by solutions of equations in some discrete space $X_{n}=\mathbf{R}^{n}$, which is not a subspace of $X$.

Example 1.1. Consider an integral equation

$$
x(t)=\int_{0}^{1} k(t, s) x(s) d s+f(t)
$$

on $C([0,1])$ and $k \in C([0,1] \times[0,1])$. Then it is natural to approximate $\int_{0}^{1} y(s) d s$ by the convergent mechanical quadrature, i.e., we write

$$
\int_{0}^{1} y(s) d s=\sum_{j=1}^{n} a_{n j} y\left(s_{n j}\right)+r_{n}(y)
$$

with fixed coefficients $a_{n j} \geq 0$, interpolation points $0 \leq s_{n 1}<\cdots<s_{n n}=1$, and the remainder $r_{n}$ such that $r_{n}(y) \rightarrow 0$ on $C([0,1])$. This is how the integral equation is approximated by the discrete system

$$
c_{n i}=\sum_{j=1}^{n} a_{n j} k\left(s_{n i}, s_{n j}\right) c_{n j}+f\left(s_{n i}\right)
$$

for $i=1, \ldots, n$, obtained by neglecting $r_{n}$ and setting $t=s_{n i}$ for $i=$ $1, \ldots, n$.

Next, we proceed to recall some definitions corresponding to approximation scheme $\pi_{1}=\left\{X_{n}, E_{n}, R_{n}, Y_{n}, Q_{n}\right\}$ represented by diagram (1.2) and the equation $A x=b \quad(x \in X)$ along with approximate equations

$$
A_{n} x_{n}=Q_{n} b \quad\left(x_{n} \in X_{n}, n=1,2, \ldots\right) .
$$

Definition 1.2 (approximation-compatibility). An approximation scheme $\pi_{1}$ is said to be compatible if for all $x \in X$

$$
\lim _{n \rightarrow \infty}\left\|E_{n} R_{n} x-x\right\|_{X}=0
$$

where $\|\cdot\|_{X}$ denotes the norm in $X$.

Definition 1.3 (admissible inner approximation scheme). An approximation scheme $\pi_{1}$ is said to be an admissible inner approximation scheme iff the following hold:

(i) $X$ and $Y$ are infinite-dimensional normed spaces over a field $\mathbf{K}$ (real or complex). 
(ii) $X_{n}$ and $Y_{n}$ are normed spaces over $\mathbf{K}$ with $\operatorname{dim} X_{n}=\operatorname{dim} Y_{n}<\infty$ for all $n$.

(iii) For all $n, E_{n}: X_{n} \rightarrow X$ and $Q_{n}: Y \rightarrow Y_{n}$ are linear and continuous with $\sup \left\|E_{n}\right\|<\infty$ and $\sup \left\|Q_{n}\right\|<\infty$.

(iv) The approximation scheme $\pi_{1}$ is compatible in the sense of Definition 1.2 .

Definition 1.4 (solvability). The equation $A x=b$ is said to be solvable if it has a solution for each $b \in Y$.

Definition 1.5 (unique approximation-solvability). The equation $A x=b$ is said to be uniquely approximation-solvable if, for each $b \in Y$, the following hold:

(i) The equation $A x=b$ has a unique solution.

(ii) For each $n \geq n_{0}$, the approximate equation $A_{n} x_{n}=Q_{n} b, x_{n} \in X_{n}$, has a unique solution.

(iii) The sequence $\left(x_{n}\right)$ converges to the solution $x$ of the equation $A x=b$ in the sense that $\lim _{n \rightarrow \infty}\left\|E_{n} x_{n}-x\right\|_{X}=0$.

Definition 1.6 (approximation-properness). A mapping $A: X \rightarrow Y$ is said to be approximation-proper (A-proper) with respect to the approximation scheme $\pi_{1}$, if the following holds: Let $\left(n^{\prime}\right)$ be any subsequence of the sequence of natural numbers. If $\left(x_{n^{\prime}}\right)$ is a sequence with $x_{n^{\prime}} \in X_{n^{\prime}}$ for all $n^{\prime}$, and if

(i) $\lim _{n \rightarrow \infty}\left\|A_{n^{\prime}} x_{n^{\prime}}-Q_{n^{\prime}} b\right\|_{Y_{n^{\prime}}}=0$ for fixed $b \in Y$ and

(ii) $\sup \left\|x_{n^{\prime}}\right\|_{X_{n^{\prime}}}<\infty$,

then there exists a subsequence $\left(x_{n^{\prime \prime}}\right)$ such that $\lim _{n \rightarrow \infty}\left\|E_{n^{\prime \prime}} x_{n^{\prime \prime}}-x\right\|_{X}=0$ and $A x=b$.

Definition 1.7 (approximation-consistency). An approximation scheme $\pi_{1}$ is said to be consistent if for all $x \in X$

$$
\lim _{n \rightarrow \infty}\left\|Q_{n} A x-A_{n} R_{n} x\right\|_{Y_{n}}=0 .
$$

Definition 1.8 (approximation-stability). An approximation scheme $\pi_{1}$ is said to be stable if for all $x, y \in X_{n}$ there is an $n_{0}$ such that

$$
\left\|A_{n} x-A_{n} y\right\|_{Y_{n}} \geq \mu\left(\|x-y\|_{X_{n}}\right)
$$

for all $n \geq n_{0}$. Here $\mu: \mathbf{R}_{+} \rightarrow \mathbf{R}_{+}$is some gauge function.

Before we proceed further, let us recall a theorem of Petryshyn [6], crucial to the development of our results on the approximation-solvability.

Lemma 1.9. If the approximation scheme $\pi_{1}=\left\{X_{n}, E_{n}, R_{n}, Y_{n}, Q_{n}\right\}$ represents an admissible inner approximation with consistency and stability, then for each $b \in Y$ the equation $A x=b, x \in X$, is uniquely approximation-solvable iff $A$ is A-proper.

Definition 1.10. Let $X$ and $Y$ be complex Banach spaces. Let a map $\phi: X \rightarrow Y$ be such that

(i) $\phi(X)$ is dense in $Y$ and

(ii) for each $x \in X$ and each $t \geq 0,\|\phi(x)\| \leq\|x\|$ and $\phi(t x)=t \phi(x)$. 
A mapping $A: X \rightarrow Y^{*}$ from $X$ into $Y^{*}$, the dual of $Y$, is said to be strongly $\phi$-stable if there is a constant $c>0$ such that

$$
|[A x-A u, \phi(x-u)]| \geq c\|x-u\|^{2} \quad \text { for all } x, u \in X,
$$

where $[\cdot, \cdot]$ is the pairing between $Y^{*}$ and $Y$.

Similarly, $A: X \rightarrow Y^{*}$ is called strongly $\phi$-monotone if there is a constant $d>0$ such that

$$
\operatorname{Re}[A x-A u, \phi(x-u)] \geq d\|x-u\|^{2} \quad \text { for all } x, u \in X .
$$

We note that when $Y=X^{*}$ ( $X$ reflexive), $A: X \rightarrow X$ is strongly $\phi$-accretive in the sense of Ray [9].

The main aim of this paper is to generalize the results on the approximationsolvability of the functional equations involving strongly stable mappings, by applying a theorem of Petryshyn [6] to the case of strongly $\phi$-stable mappingsa new and rather general class of mappings-in a Hilbert space setting. In the final section, we upgrade the obtained results to a Banach space setting with a more general approximation scheme.

A word of caution: For the sake of simplicity, in what follows the symbol $\|\cdot\|$ shall denote all norms $\|\cdot\|_{X},\|\cdot\|_{X_{n}},\|\cdot\|_{Y},\|\cdot\|_{Y_{n}},\|\cdot\|_{Y^{*}}$, and $\|\cdot\|_{Y_{n}^{*}}$ in spaces $X, X_{n}, Y, Y_{n}, Y^{*}$, and $Y_{n}^{*}$, respectively. We feel the steps will be self-indicative to the reader of which norm is used.

\section{INNER APPROXIMATION SCHEME}

Let $X$ be a separable complex Hilbert space with $\operatorname{dim} X=\infty$. Let us consider the equation

$$
A x=b, \quad x \in X,
$$

along with approximate equations

$$
P_{n} A E_{n} x_{n}=P_{n} b
$$

for $x_{n} \in X_{n}, n=1,2, \ldots$, corresponding to an approximation scheme $\pi_{2}=$ $\left\{X_{n}, E_{n}, P_{n}\right\}$, represented by a diagram

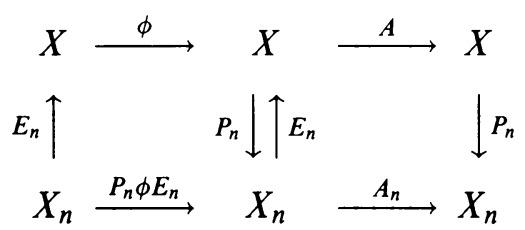

where all $A_{n}=P_{n} A E_{n}$ are continuous, $X_{n}=\operatorname{span}\left\{e_{1}, \ldots, e_{n}\right\}$ for a complete orthonormal system $\left(e_{i}\right)$ in $X, P_{n}: X \rightarrow X_{n}$ is orthogonal projection from $X$ onto $X_{n}$ and $E_{n}: X_{n} \rightarrow X$ is the embedding with $X_{n} \subseteq X$ (i.e., $E_{n} x_{n}=x_{n}$ for all $x_{n} \in X_{n}$ ). Then the approximate problems (2.2) relate to a projection method-Galerkin method. For $n=1,2, \ldots$, equation (2.2) is equivalent to the Galerkin equations

$$
\left\langle A x_{n}, \phi e_{j}\right\rangle=\left\langle b, \phi e_{j}\right\rangle
$$

for $x_{n} \in X_{n}, j=1, \ldots, n$, where $\langle\cdot, \cdot\rangle$ is the standard inner product. Note that $E_{n}$ and $P_{n}$ are linear and continuous for all $n$. 


\section{SOLVABILITY AND APPROXIMATION-SOLVABILITY}

Let us first start with the solvability of the equation $A x=b$.

Theorem 3.1. Suppose $A: X \rightarrow X$ is strongly $\phi$-stable and continuous on $a$ separable Hilbert space $X$ with $\operatorname{dim} X<\infty$ and that $\phi: X \rightarrow X$ is continuous. Then the equation $A x=b \quad(x \in X)$ has a unique solution for each $b \in X$.

For $\phi=I$ in Theorem 3.1, we have

Corollary 3.2. If $A: X \rightarrow X$ is strongly stable, then the equation $A x=b \quad(x \in$ $X)$ has a unique solution for every $b \in X$.

Proof of Theorem 3.1. Since $A$ is strongly $\phi$-stable, it is immediate that there is a constant $c>0$ such that

$$
|\langle A x-A y, \phi(x-y)\rangle| \geq c\|x-y\|^{2}
$$

for all $x, y \in X$. It follows that

$$
\|A x-A y\| \geq c\|x-y\|,
$$

and so $A$ is injective. Furthermore, by using condition (3.1), we have for $x \in X$

$$
\begin{aligned}
|\langle A x, \phi(x)\rangle| & =|\langle A x-A(0), \phi(x)\rangle+\langle A(0), \phi(x)\rangle| \\
& \geq|\langle A x-A(0), \phi(x)\rangle|-|\langle A(0), \phi(x)\rangle| \\
& \geq c\|x\|^{2}-\|A(0)\|\|\phi(x)\| \\
& \geq c\|x\|^{2}-\|A(0)\|\|x\|=\psi(\|x\|)\|x\|,
\end{aligned}
$$

where $\psi(r)=c r-\|A(0)\|$ is some gauge function. It follows that $\|A x\| \geq$ $\psi(\|x\|)$ for $x \neq 0$. For each $M>0$, therefore, there exists $k(M)$ such that $\|A x\| \leq M$, implying $\|x\| \leq k(M)$. Thus, $A^{-1}$ carries bounded subsets of $R(A)$, the range of $A$, into bounded subsets of $X$, and is Lipschitz continuous from $R(A)$ to $X$. By the Brouwer theorem on invariance of domain, $R(A)$ is open. To this end, it only remains to show that $R(A)$ is closed.

To show this, let $A x_{m} \rightarrow b$ as $m \rightarrow \infty$. Thus $\left(A x_{m}\right)$ is a Cauchy sequence. By condition (3.1), we have

$$
\begin{aligned}
c\left\|x_{m}-x_{n}\right\|^{2} & \leq\left|\left\langle A x_{m}-A x_{n}, \phi\left(x_{m}-x_{n}\right)\right\rangle\right| \\
& \leq\left\|A x_{m}-A x_{n}\right\|\left\|\phi\left(x_{m}-x_{n}\right)\right\| \leq\left\|A x_{m}-A x_{n}\right\|\left\|x_{m}-x_{n}\right\|,
\end{aligned}
$$

and so

$$
c\left\|x_{m}-x_{n}\right\| \leq\left\|A x_{m}-A x_{n}\right\| \rightarrow 0 \quad \text { as } m, n \rightarrow \infty .
$$

It follows that $\left\|x_{m}-x_{n}\right\| \rightarrow 0$, and hence $\left(x_{m}\right)$ is also a Cauchy sequence, implying $x_{m} \rightarrow x$ as $m \rightarrow \infty$. Since $A$ is continuous, $A x=b$, implying $b \in R(A)$. This concludes the proof.

Theorem 3.3. Let $\pi_{2}=\left\{X_{n}, E_{n}, P_{n}\right\}$ be the approximation scheme represented by diagram (2.3), and let $\phi: X \rightarrow X$ be such that

$$
P_{n} \phi E_{n} x=\phi x \quad \text { for all } x \in X_{n} \text { and each } n \text {. }
$$

If $A: X \rightarrow X$ is strongly $\phi$-stable and continuous on a separable Hilbert space $X$ with $\operatorname{dim} X=\infty$, and if $\phi$ is weakly continuous, then the equation $A x=b$ $(x \in X)$ is uniquely approximation-solvable for each $b \in X$. 
Corollary 3.4. In addition to the assumptions of Theorem 3.3, if $B: X \rightarrow X$ is compact then $A+B: X \rightarrow X$ is also A-proper with respect to the approximation scheme $\pi_{2}$.

Corollary 3.5. Let $\pi_{2}=\left\{X_{n}, E_{n}, P_{n}\right\}$ be the approximation scheme. If $A: X \rightarrow$ $X$ is strongly $\phi$-monotone and continuous, and if $B: X \rightarrow X$ is compact, then for each nonzero $\lambda \in \mathbf{K}$ (field), the mapping $\lambda(A+B): X \rightarrow X$ is $A$-proper with respect to approximation scheme $\pi_{2}$.

Corollary 3.6. For $\phi=I$, Theorem 3.3 is reduced to Zeidler [15, Theorem 34.B]. Proof of Theorem 3.3. To prove this, we apply Lemma 1.9, i.e., we first need to show the approximation scheme $\pi_{2}$ is an admissible inner approximation scheme along with consistency and stability and then $A$ is A-proper with respect to $\pi_{2}$.

Since $\left\|E_{n}\right\|=1$ and $\left\|P_{n}\right\|=1$ For all $n$, and since $P_{n} x \rightarrow x$ as $n \rightarrow \infty$ for all $x \in X$, this implies that

$$
\lim _{n \rightarrow \infty}\left\|E_{n} P_{n} x-x\right\|=0 \quad \text { for all } x \in X,
$$

i.e., the compatibility condition, in the sense of Definition 1.2.

Consistency. Since the approximation scheme $\pi_{2}$ is compatible and $A$ is continuous, it follows that $\left\|A E_{n} P_{n} x-A x\right\| \rightarrow 0$ as $n \rightarrow \infty$. Furthermore, since $\sup \left\|P_{n}\right\|<\infty$, we arrive at the consistency condition for all $x \in X$

$$
\begin{aligned}
\left\|P_{n} A x-A_{n} P_{n} x\right\| & =\left\|P_{n} A x-P_{n} A E_{n} P_{n} x\right\| \\
& \leq\left\|P_{n}\right\|\left\|A x-A E_{n} P_{n} x\right\| \rightarrow 0 \quad \text { as } n \rightarrow \infty .
\end{aligned}
$$

Stability. Since $A$ is strongly $\phi$-stable, we have for all $x, y \in X$

$$
|\langle A x-A y, \phi(x-y)\rangle| \geq c\|x-y\|^{2} \text {. }
$$

For all $x, y \in X_{n}$, it follows that

$$
\begin{aligned}
\left\|A_{n} x-A_{n} y\right\|\|\phi(x-y)\| & \geq\left|\left\langle A_{n} x-A_{n} y, \phi(x-y)\right\rangle\right| \\
& =\left|\left\langle P_{n} A E_{n} x-P_{n} A E_{n} y, \phi(x-y)\right\rangle\right| \\
& =\left|\left\langle A x-A y, P_{n} \phi(x-y)\right\rangle\right| \\
& =|\langle A x-A y, \phi(x-y)\rangle| \geq c\|x-y\|^{2},
\end{aligned}
$$

and consequently,

$$
\left\|A_{n} x-A_{n} y\right\| \geq c\|x-y\| \text { for all } x, y \in X_{n} .
$$

A-properness. To show $A$ is A-proper, let $\sup \left\|x_{n}\right\|<\infty$ and

$$
\left\|P_{n} A E_{n} x_{n}-P_{n} b\right\| \rightarrow 0 \quad \text { as } n \rightarrow \infty,
$$

where $x_{n} \in X_{n}$. Since $X$ is reflexive, there exists a subsequence (again) denoted $\left(x_{n}\right)$ such that $x_{n} \rightarrow x$ in $X$ as $n \rightarrow \infty$. We need only show that $x_{n} \rightarrow x$ in $X$ as $n \rightarrow \infty$, and $A x=b$. For all $y \in X$, we have

$$
\left\langle x_{n}-P_{n} x, y\right\rangle=\left\langle x_{n}, y\right\rangle-\left\langle P_{n} x, y\right\rangle \rightarrow 0,
$$

and so $x_{n}-P_{n} x \rightarrow 0$. It follows that, as $n \rightarrow \infty$,

$$
P_{n} b \rightarrow b, \quad P_{n} x \rightarrow x, \quad A P_{n} x \rightarrow A x, \quad P_{n} A P_{n} x \rightarrow A x,
$$


and hence

$$
P_{n}\left(A x_{n}-A P_{n} x\right)=\left[P_{n} A x_{n}-P_{n} b\right]+\left[P_{n} b-P_{n} A P_{n} x\right] \rightarrow b-A x .
$$

Now, by condition (3.1), we have

$$
\begin{aligned}
c\left\|x_{n}-P_{n} x\right\|^{2} & \leq\left|\left\langle P_{n} A x_{n}-P_{n} A P_{n} x, \phi\left(x_{n}-P_{n} x\right)\right\rangle\right| \\
& =\left|\left\langle P_{n}\left(A x_{n}-A P_{n} x\right), \phi\left(x_{n}-P_{n} x\right)\right\rangle\right| \rightarrow 0 \quad \text { as } n \rightarrow \infty,
\end{aligned}
$$

and so $\left\|x_{n}-P_{n} x\right\| \rightarrow 0$ as $n \rightarrow \infty$, implying $x_{n}-P_{n} x \rightarrow 0$ as $n \rightarrow \infty$. Since $\left\|E_{n}\right\|<\infty$, it implies

$$
\left\|E_{n} x_{n}-E_{n} P_{n} x\right\| \rightarrow 0 \quad \text { as } n \rightarrow \infty .
$$

Moreover, the compatibility condition (in the sense of Definition 1.2) implies

$$
\left\|E_{n} P_{n} x-x\right\| \rightarrow 0 \quad \text { as } n \rightarrow \infty \text {. }
$$

It follows that $\left\|E_{n} x_{n}-x\right\| \rightarrow 0$ as $n \rightarrow \infty$. Again, since $x_{n}-P_{n} x \rightarrow 0$ implies that $x_{n} \rightarrow x$ as $n \rightarrow \infty$, the assumption (3.3) implies that $A x_{n} \rightarrow b$, and $A$ is continuous, we obtain $A x=b$, i.e., $A$ is A-proper.

Now the proof follows by an application of Lemma 1.9, and this concludes the proof.

\section{CONCLUDING REMARKS}

It seems that Theorem 3.3 can be upgraded to the case of Banach spaces by extending the approximation scheme in the following manner: Let $X$ and $Y$ be separable complex Banach spaces ( $X$ reflexive) with $\operatorname{dim} X=\operatorname{dim} Y=\infty$, and let $A: X \rightarrow Y^{*}$ from $X$ to $Y^{*}$, the dual of $Y$, be strongly $\phi$-stable and continuous. Let $\left(X_{n}\right)$ and $\left(Y_{n}\right)$ respectively be Galerkin schemes in $X$ and $Y$ such that

$$
X_{n}=\operatorname{span}\left\{e_{1 n}, \ldots, e_{n^{\prime} n}\right\}
$$

and

$$
Y_{n}=\operatorname{span}\left\{f_{1 n}, \ldots, f_{n^{\prime} n}\right\}, \quad n=1,2, \ldots .
$$

Let $E_{n}: X_{n} \rightarrow X$ and $Q_{n}: Y_{n} \rightarrow Y$ be the embedding mappings with $X_{n} \subseteq X$ and $Y_{n} \subseteq Y$. We construct $R_{n}: X \rightarrow X_{n}$ in such a manner that for each $x \in X$ there exists at least an element $R_{n} x \in X_{n}$ such that $\left\|x-R_{n} x\right\|=\operatorname{dist}\left(x, X_{n}\right)$.

We consider the equation

$$
A x=b, \quad x \in X, b \in Y^{*},
$$

along with approximation equations

$$
A_{n} x_{n}=Q_{n}^{*} b, \quad x_{n} \in X_{n}, n=1,2, \ldots,
$$

corresponding to an approximation scheme $\pi_{3}=\left\{X_{n}, E_{n}, R_{n}, Y_{n}, Q_{n}, S_{n}\right.$, $\left.Y_{n}^{*}, Q_{n}^{*}\right\}$ represented by a diagram

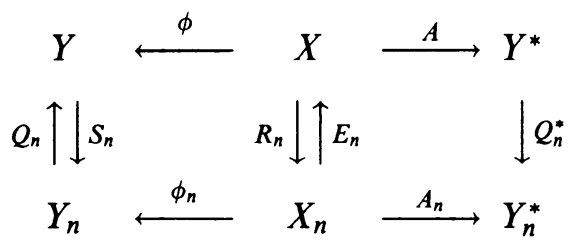


where $\phi_{n}=S_{n} \phi E_{n}$ and $A_{n}=Q_{n}^{*} A E_{n}$ are continuous and $E_{n}, Q_{n}, Q_{n}^{*}, S_{n}$ are linear and continuous.

For $n=1,2, \ldots$, the approximate equation (4.2) is equivalent to

$$
\left[A x_{n}, S_{n} \phi e_{j n}\right]=\left[b, S_{n} \phi e_{j n}\right]
$$

for $x_{n} \in X_{n}, j=1, \ldots, n^{\prime}$.

Theorem 4.1. Let $\pi_{3}=\left\{X_{n}, E_{n}, R_{n}, Y_{n}, S_{n}, Q_{n}, Y_{n}^{*}, Q_{n}^{*}\right\}$ be an approximation scheme represented by diagram (4.3), and let $\phi: X \rightarrow Y$ be such that

$$
S_{n} \phi E_{n} x=\phi x \quad \text { for all } x \in X_{n} \text { and each } n \text {. }
$$

If $A: X \rightarrow Y^{*}$ is strongly $\phi$-stable and continuous, and if $\phi$ is weakly continuous, then for each $b \in Y^{*}$ the equation $A x=b, x \in X$, is uniquely approximation-solvable.

For $Y=X, Y_{n}=X_{n}, S_{n}=R_{n}, Q_{n}=E_{n}$, and $Q_{n}^{*}=E_{n}^{*}$, diagram (4.3) reduces to

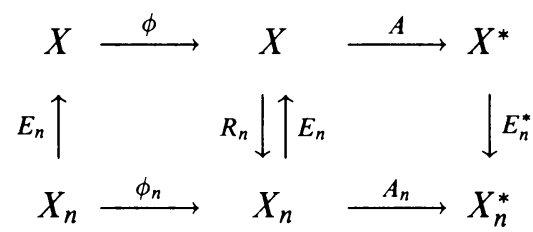

corresponding to an approximation scheme $\pi_{4}=\left\{X_{n}, E_{n}, R_{n}, X_{n}^{*}, E_{n}^{*}\right\}$.

Corollary 4.2. Let $\pi_{4}=\left\{X_{n}, E_{n}, R_{n}, X_{n}^{*}, E_{n}^{*}\right\}$ be an approximation scheme for the pair $\left(X, X^{*}\right)$, and let $\phi: X \rightarrow X$ be such that

$$
R_{n} \phi E_{n} x=\phi x \quad \text { for all } x \in X_{n} \text { and each } n \text {. }
$$

If $A: X \rightarrow X^{*}$ is strongly $\phi$-stable and continuous, then for each $b \in X^{*}$ the equation $A x=b, x \in X$, is uniquely approximation-solvable.

Corollary 4.3. For $\phi=I$ in Corollary 4.2, we arrive at Zeidler [15, Proposition 34.9] corresponding to an approximation scheme represented by

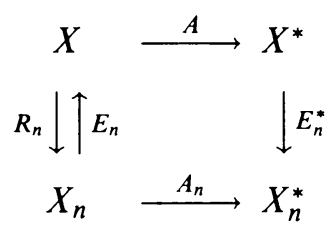

\section{REFERENCES}

1. F. Browder and W. Petryshyn, Approximation methods and the generalized topological degree for nonlinear mappings in Banach spaces, J. Funct. Anal. 3 (1969), 217-245.

2. K. Deimling, Nonlinear functional analysis, Springer-Verlag, New York, 1985.

3. A. G. Kartsatos, On the equation $T x=y$ in Banach spaces with weakly continuous duality maps, Nonlinear Equations in Abstract Spaces, Academic Press, New York, 1978.

4. M. A. Krasnosel'skii, G. M. Vainikko, P. P. Zabreiko, Ya. B. Rutitskii, and V. Ya. Stetsenko, Approximation solution of operator equations, English transl., Wolter-Noordhoff, Groningen, 1972.

5. R. N. Mukherjee and T. Som, On phi-monotone operators and variational inequalities, Bull. Calcutta Math. Soc. 80 (1988), 201-205. 
6. W. Petryshyn, Nonlinear equations involving noncompact operators, Nonlinear Functional Analysis (Chicago, 1968), Proc. Sympos. Pure Math., vol. 18, part 1, Amer. Math. Soc., Providence, RI, 1970, pp. 206-233.

7. __, On the approximation-solvability of equations involving A-proper and pseudo-A-proper mappings, Bull. Amer. Math. Soc. 81 (1975), 223-312.

8. __ Approximation-solvability of periodic boundary value problems via the A-proper mapping theory, Proc. Sympos. Pure Math. vol. 45, Amer. Math. Soc., Providence, RI., 1986, pp. 261-282.

9. W. O. Ray, Phi-accretive operators and Ekeland's theorem, J. Math. Anal. Appl. 88 (1982), 566-571.

10. I. G. Rosen, Convergence of Galerkin approximation for operators Riccati equations-A nonlinear evolution equation approach, J. Math. Anal. Appl. 155 (1991), 226-248.

11. M. Théra, Existence results for the nonlinear complementarity problem and applications to nonlinear analysis, J. Math. Anal. Appl. 154 (1991), 572-584.

12. V. B. Trushin, On the solution of certain nonlinear equations and variational inequalities, Soviet Math. Dokl. 40 (1990), 521-524.

13. R. Verma, Role of numerical range in approximation-solvability of nonlinear functional equations, Appl. Math. Lett. 5 (1992), 25-27.

14. R. Verma and R. Mohapatra, Application of numerical range to approximation-solvability of nonlinear functional equations, PanAmerican Math. J. 1 (1991), 46-54.

15. E. Zeidler, Nonlinear functional analysis and its applications II/B: Nonlinear monotone operators, Springer-Verlag, Berlin and New York, 1990.

Department of Mathematics, University of Central Florida, Orlando, Florida 32816 